\title{
Design and Implementation of e-mail Client Application
}

\author{
Hongqing Liu \\ Hunan vocational college of modern logistics,Changsha, Hunan,410131
}

Keywords: Mail, Application program, Mail system, System design, Office automation.

\begin{abstract}
E-mail is a kind of communication mode which provides information exchange through electronic means. Nowadays, more and more enterprise units cooperate with e-mail to break the limitation of time and domain, and realize office automation, which greatly improves work efficiency. It can be said that e-mail has become an indispensable part of people's life. This paper first describes the development background and system development platform of e-mail client application, and introduces the technology used in the system development. In the analysis phase of the system, the structure of e-mail and the format of e-mail text are analyzed, the working principle of e-mail is mastered, and the SMTP and POP3 protocols are deeply studied. In the design and implementation of the system, determine the system development ideas and development environment. Finally, the detailed design and implementation of the module in the system are given, and the key source code involved in the system module is analyzed in detail.
\end{abstract}

\section{Introduction}

E-mail (E-mai1) is also called electronic mail box. Electronic mail is a kind of communication mode which provides information exchange by electronic means. It is the most common service used in many kinds of networks all over the world. This non interactive communication accelerates the exchange of information and data transmission, and it is a simple and fast method. Through the connection of the world's Internet, to achieve all kinds of signal transmission, receiving, storage and other processing, mail to the world in every corner. So far, can be said that e-mail is the most used Internet resources as a service, E-mai1 not limited to letters of transfer, can also be used to transfer files, voice and graphics, images of different types of information [1].

E-mail is not a "end-to-end" service, which is called "storage and forwarding" service. This is the core of the electronic mailbox system, which can be used for asynchronous communication by using storage and forwarding for non real time communication. That is, the sender can send mail anytime and anywhere, without requiring the receiver to be present. Even if the party is not available, the message can be sent to the mailbox of the other party immediately and stored in the e-mail address of the other party. In the receiver that is convenient when reading the letters, not limited by time and space here, send mail to the recipient's mailbox will mean the mail, and receive mail means read letters from your mailbox, the mailbox is actually an entity supported by the file management system. Because e-mail is passed through the mai1 server to deliver documents. Usually mail server UNIX operating system of multi mission computer, it provides 24 hours of e-mail service, as long as the user to the mail server management personnel to apply for a mail account, you can use the fast mail service [2].

\section{Working Principle}

POP3(Post Office Protocol 3) It is an offline model email protocol for C/S structure, which has now been developed to version third. POP3 specifies how to connect a personal computer to an Internet mail server and Download E-mail rules. POP3 is the first offline protocol standard Internet email, allowing users from the server to the mail storage to the local host (i.e. your computer), and delete preserved in email messages on the server. POP3 server is a receiving mail server following 
POP3 protocol, which is used to receive e-mail. POP is suitable for offline models of C/S structures, and offline models cannot operate online (unlike IMAP4 when clients connect to the server and query new e-mail). All customers machine specified will be downloaded messages will be downloaded to the client, download, email client can delete or modify any mail, without further interaction with the email server. The POP3 client sends a command to the POP3 server and waits for the response. The POP3 command is in the command line format and is represented by the ASCII code. The server response is composed of a single component or a plurality of the command line, the command line, in response to the first line to ASCII +OK or -ERR text pointed out that the operating state of the corresponding success or failure has three states, in the POP3 protocol approved by state, the processing state, and update the status. When connect the client and server, once the client provides its own identity and successfully confirmed by authorized state to state, issue the quit command to complete the corresponding operation in the client, enter the status update, update after the last return to the approval status.

Most of the existing POP3 clients and servers use ASCII plaintext to send commands, the customer sends the connection, and sends the plaintext user name and password to the server to confirm the identity by the command user/pass. Once the validation is successful, it is transferred to the processing state. In order to avoid sending plaintext password, there is a new authentication method, the command is APOP, using APOP, the password is encrypted before transmission. When the server is connected for the first time, the POP3 server sends a ASCII code greeting to the client. The greeting is composed of a string of characters, unique to each client, and related to the time at that time. Then, after the client to plain text password it attached to a string received from the server, and then calculate the result string MD5 single function message digest, the client user name and MD5 message as the order parameter APOP sent out together. Currently, most mail client software on Windows does not support APOP commands.

\section{3 . System Design}

The process of the system is divided into the following 7 steps:

1. registered users

E-mail is forwarded and charged through the network mail server, so it must be registered on the network mail server to send and receive e-mail and attachments. When the registered user fails, login again; if successful, log on to the network mail server and execute the next step.

2. send mail

When the login server is successful, the user can send e-mail and its attachments from the server, if not successful, resend; if successful download, then the next step is executed.

\section{3. collect mail}

When the login server is successful, the user can download the e-mail and its attachments from the server. If unsuccessful, it will be downloaded again; if it is downloaded successfully, the next step will be executed.

4. open mail

When the user downloads the e-mail on the network mail server to the local computer, it can open the mail and display the relevant information (title, sender, recipient, text) of the mail. And open it in accordance with the corresponding format of the attachment (DOC, JPEG, etc.).

5. save mail

When the user downloads the e-mail on the network mail server to the local computer, the mail can be saved. Save the relevant information (title, sender, recipient, text) in the mail storage directory of the machine and save it as a TXT document format. And the corresponding accessories can be saved in this directory. 


\section{6. delete mail}

When the user downloads the e-mail on the network mail server to the local computer and saves the mail. You can delete the mail in the mail storage directory, and delete the attachments.

\section{7. exit system}

After the user completes the corresponding operation, quit the system. Such as: caitian6 account registration and login to the mail server, after receiving mail, mail is stored in the local directory. Then send mail to friends, save the mail in the local directory. When the sending and receiving letters are closed, exit the system.

In the account management case, there are 4 actions, which are as follows:

Account management: access to account management, you can create new users in the network mail server, modify the user, delete users.

New users: set up the SMTP server address, set the POP3 server address, set the user name, set the password on the machine. Also create users on the web mail server.

Modify the user: modify the SMTP server address on this machine, modify the POP3 server address, modify the user name, modify the password. Also modify the user on the network mail server.

Delete user: modify the SMTP server address on this machine, modify the POP3 server address, modify the user name, modify the password. At the same time, delete users on the network mail server.

1. get the current directory

Call the GetModuleFileName function to get the current directory and save it in the string sPath.

2. create save directory folder

After the current directory string sPath, append the "file directory" character, and call the SetCurrentDirectory function to determine whether the saved file directory exists. If it does not exist, call CreateDirectory to create the directory in the current directory.

3. create save file dialog box

Create the object DLG of the CfileDialog class, and the DLG is the save file dialog box. Set the dialog box titled "save the message in", and set the default path of the dialog box to sPath. Call the DoModal function to display the dialog box.

4. create file objects

Create the object $\mathrm{F}$ of the CFile class, set the path of the file object to the current directory, and create it in that directory

Save the txt file of the mail.

5. save mail content

Create the object AR1 of the CArchive class. The object is accessed by CArchive:: store. The mail content strTemp is stored in AR1, that is, the content of the mail is stored in the TXT file.

\section{Summary}

This paper expounds the development and significance of e-mail, analyzes the current development trend and existing problems of e-mail in China, and advances the rapid development of computer, electronic technology and communication, speeding up the development of e-mail technology. In the requirement analysis phase, after in-depth study of SMTP, POP3 protocol, master SMTP, POP3 communication model and working principle, SMTP, POP3 command and its 
response. In the detailed design and coding phase, the WINSOCKET technology is used to make the code clear and easy to understand. The system structure is clear, designed to send mail class Smailer, receive mail class Cpop3. On the basis of the two types, the functions of sending mails, receiving mails and managing mails are completed.

\section{References}

[1]Dalia S. Abdelhamid, Yingyue Zhang, Daniel R. Lewis, Prabhas V. Moghe, William J. Welsh, and Kathryn E. Uhrich, Tartaric Acid-based Amphiphilic Macromolecules with Ether Linkages Exhibit Enhanced Repression of Oxidized Low Density Lipoprotein Uptake, Biomaterials, 2015, 53, 32-39.

[2]Yingyue Zhang, Jennifer W. Chan, AlyshaMoretti, and Kathryn E. Uhrich, Designing Polymers with Sugar-based Advantages for Bioactive Delivery Applications, Journal of Controlled Release, 2015, 219, 355-368.

[3]Yingyue Zhang, Qi Li, William J. Welsh, Prabhas V. Moghe, and Kathryn E. Uhrich, Micellar and Structural Stability of NanoscaleAmphiphilic Polymers: Implications for Anti-atherosclerotic Bioactivity, Biomaterials, 2016, 84, 230-240. 